\title{
The development of artificial intelligence in the field of recommendation system
}

\author{
Liyang Yan ${ }^{1, a}$, Hui $\mathrm{CaO}^{2, \mathrm{~b}}$ and Xiangzhen $\mathrm{He}^{3, \mathrm{c},{ }^{*}}$ \\ ${ }^{1}$ Key Laboratory of China's Ethnic Languages and Information Technology of Ministry of \\ Education, Northwest Minzu University, Lanzhou, Gansu 730000, China \\ ${ }^{2}$ Key Laboratory of China's Ethnic Languages and Information Technology of Ministry of \\ Education, Northwest Minzu University, Lanzhou, Gansu 730000, China \\ ${ }^{3}$ Key Laboratory of China's Ethnic Languages and Information Technology of Ministry of \\ Education, Northwest Minzu University, Lanzhou, Gansu 730000, China \\ ayanliyang0821@qq.com, b147625251@qq.com, chexiangzhen@163.com \\ * Xiangzhen $\mathrm{He}$
}

Keywords: Recommendation system, Artificial intelligence, Deep learning, Collaborative filtering.

\begin{abstract}
In recent years, with the rise of artificial intelligence, great progress has been made in computer vision, speech recognition and natural language processing. In the rapid change of technology, the recommendation system has become an important part of many Internet applications. The development of nearly 10 studies has made this field rapidly emerging, and the recommendation system based on deep learning has become a research hotspot in the industry.
\end{abstract}

\section{The introduction}

Artificial intelligence has become a hot topic in IT industry in recent years. It has been continuously promoted, especially in the fields of computer vision, speech recognition and natural language processing. With the rapid development of the Internet industry, the access to information is increasing, and the speed of data generation is multiplied. For example, News subscribed by RSS will have thousands of messages to read in a matter of days; WeChat public number, for a period of time not to read also has a lot of unread information. Massive information makes it difficult for people to obtain effective information. In order to cope with information overload, a more effective and targeted information system for obtaining the information it needs has produced a recommendation system. Linking artificial intelligence with recommendation systems has become a hot topic.

\section{Overview of artificial intelligence.}

The word "artificial intelligence" must have been heard by most people and it is easy to understand. Artificial intelligence is the science and engineering that enables machines and programs to learn and solve problems in a way that usually requires human intelligence. In simple terms, artificial intelligence is a machine designed by humans to approximate or even surpass human's in natural language, visual perception and thinking analysis. The biggest difference between artificial intelligence and human is storage capacity, computational speed,energy and energy. Machines have the upper edge, but no human intelligence, so the combination of the two is called artificial intelligence.

Artificial intelligence, machine learning and deep learning involve relationships. The biggest difference between machine learning and previous computer programs is that things that need to be resolved by the machine are not entered in advance by the machine, but are learned by the machine itself, which has a qualitative leap compared to the previous one. Deep learning is designed for the computer to simulate the structure of the human neural network. Simply put, a different thing is marked by different things at different levels, which can solve all kinds of complex problems. For 
example, in order to identify a goat image, then the underlying object will identify whether it has horns, if the answer is certain, the next layer will be looking for wool, the next step will be looking for face and so on, until all the layers to determine the image as a goat or rejected.

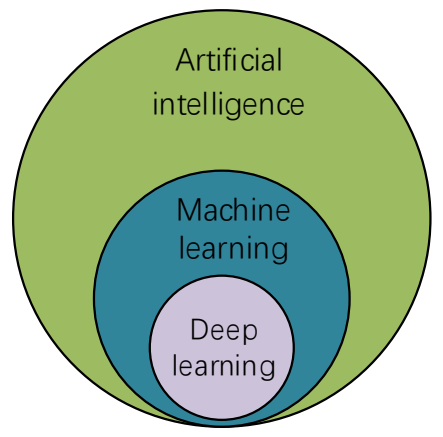

Fig. 1. Diagram of artificial intelligence, machine learning and deep learning.

Artificial intelligence is a business-oriented, multi-dimensional, multidisciplinary concept. The core is: algorithms, computing power and data. Based on a large number of data, the data is characterized, and a useful algorithm model is constructed. Then, based on distributed computing power, the algorithm model is applied in the production environment, so that the machine can replace the artificial to achieve the goal of improving efficiency.

How do people think of using deep learning to process recommendation systems? The development of recommender system has experienced from the content recommendation to collaborative filtering recommendation, from the collaborative filtering of users and item to the collaborative filtering of the base model. The effect of deep learning in the recommender system is not as good as image processing and speech processing, However, deep learning has enlightening significance for the recommendation system research. Look at the advantages of deep learning in the recommendation system.

Table 1. reasons for using deep learning in the recommended system.

\begin{tabular}{|l|}
\hline Extract features directly from the content, strong ability to characterize \\
\hline Easy to process noise data, strong noise immunity \\
\hline Modeling Dynamic or Sequence Data Using RNN Recurrent Neural Networks \\
\hline Learn the characteristics of user and item accurately. \\
\hline Facilitate the unified processing of responsible data. \\
\hline
\end{tabular}

In recent years, many of its companies have conducted research on deep learning and further improved the quality of recommendations. For example, YouTube uses a deep neural network based video recommendation algorithm. Shumpei and others have introduced the RNN based news recommendation system for yahoo news. These models have undergone on-line testing and have shown significant improvements over conventional models. From this we can see the changes that deep learning brings to the recommended application areas.

\section{Recommendation system overview}

\subsection{The way to obtain information}

From the perspective of information acquisition, search and recommendation are the main means for users to obtain information. Search and recommendation coexist in both the Internet and offline scenes. Whether it is search or recommendation, they are a mechanism of information acquisition, the main difference is active acquisition and passive acquisition. 


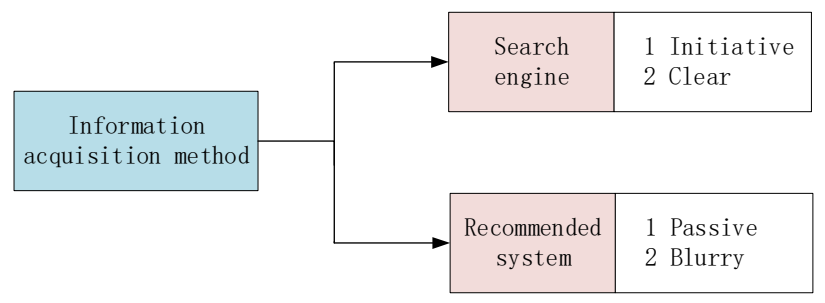

Fig.2.Shows two different ways to get information.

The search engine is a typical form of active trigger, that is, the user has clear information to obtain the intention, and he is eager to get the target information he needs, and then finally obtains the information through the search. The recommendation system is different, it is a system active behavior, the user passively accepts the information that the system pushes. Therefore, the focus of the recommendation system is to solve the user's problems of obtaining additional information. It is recommended to solve the problems of massive information redundancy and help the user to determine the goal if the purpose is not clear.

\subsection{Recommendation system}

With the rapid development of e-commerce, personalized news, and social media, discovering user's needs, understanding user's behavior, and screening more effective information and products for users are the core of Internet services. Recommender systems play an important role in helping users find information to help them find customers. On the one hand, the recommendation system has become the main business of large sites such as Baidu, Taobao, Today's headlines, and Netflix, providing personalized services for user and system interactions. It's hard to imagine an Internet application that doesn't use a recommendation system to optimize the user's experience. On the other hand, from the early collaborative filtering algorithms to the implicit semantic models promoted by the Netflix competition to the more complex deep learning models, the technology of the recommendation system has made great progress in the past decade or more. The ultimate goal of the recommender system is to accurately predict the user's preferences and gradually evolve into a balance between users, modules and systems, so as to make the whole system run healthily.

The recommender system is a very complex system, involving data processing, structure system, recommendation logic, data feedback recovery, effect tracking, AB test, etc. Good recommendation system can effectively improve user's experience, better retain users, and further enhance the profit of goods. For some e-commerce products, personalized recommendations can help reduce the effects of the Matthew effect and the long-tail effect, so that the utilization rate of the goods is higher and the profitability is increased. 


\subsection{Common recommendation algorithms.}

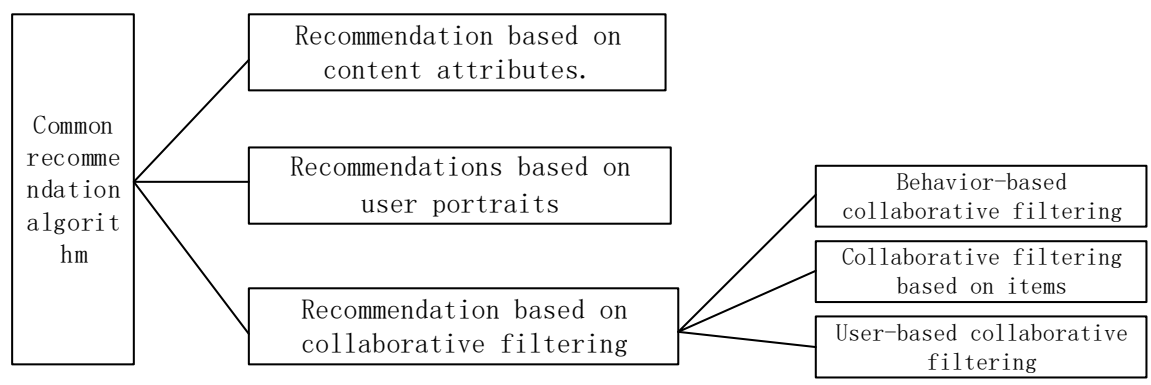

Fig. 3.Common recommendation system algorithm.

\subsubsection{Recommendation based on content attributes.}

From the perspective of the original data dependence, the recommendation logic based on the content attribute recommendation mechanism is very simple. It is simply to rely on the attribute similarity between the items to construct the recommendation relationship. It is more effective to deal with relationships that are not particularly complicated.

\subsubsection{Recommendation based on user portrait.}

The recommendation based on the user's portrait (also referred to as user's tab) mainly depends on the user's portrait attribute, and the user's preference information is used to select the candidate set according to the preference. This is a common practice and the most commonly used mechanism in the case of large-scale data sets. How does the user's portrait or user's interest tag be constructed? It mainly relies on the user's accumulated behavior data to generate the user's interest tag through the behavior data. But in general, users' interests will change over time. Therefore, it is not easy to grasp the user's interest and change, not to mention the actual choice of the user will be affected by many factors, although it is still very effective to make recommendations based on the user's preferences.

\subsubsection{Recommendation based on collaborative filtering.}

Collaborative filtering properties research items itself, not also didn't go to build the user's portrait, as its literal meaning, to a large extent depends on the user behavior and its surrounding collaborative behavior of users. For a user to recommend information, I only need to refer to what the user is looking at, so I can recommend something to him. It focused on, around how to define the scope, for instance, according to the behavior of the two users to build relationship, thus to judge the similarity degree between the users, to recommend user's behavior similar to the current user, this is the synergy of classic based on user's recommendation.

If you use the item as a dimension and you use the user's purchase or viewing record as a vector, you can construct a similar measure for the item. For each option to be recommended, the user's historical track is its vector composition, and you can determine the user history track information. Relevance with the vector of the current item to be selected, so as to judge whether to recommend, this is based on the cooperation of the item.

At present, recommendation based on collaborative behaviors, besides based on items and based on users, there are also other recommendation mechanisms such as model-based collaboration, typically such as nearest-neighbor models, matrix-based decomposition, and the construction of graph-based relational models.

\section{Deep learning in the recommendation system application vision.}

In 2006, begin to deep learning had a disruptive influence on the whole field of artificial intelligence, many scholars and enterprises begin to try to use deep learning model to solve the traditional model 
is difficult to solve problems that can't even be solved. In the past four years, deep learning has developed rapidly in fields such as natural language processing and image processing. In addition, it has also developed rapidly in the field of reinforcement learning and recommendation system. The exploration of using deep learning in the recommendation system is divided into two directions.

The first is to replace traditional models with deep learning models to improve the recommended effects of known problems, such as whether to replace matrix factorizations with deep learning models. The newer representatives, such as Hao Wang of the Hong Kong University of science and technology and Yao $\mathrm{Wu}$ of Simon Fraser University, have tried to expand the scale of the autoencoder to improve the accuracy of the scoring prediction. The deep learning technique is used to extract the high level features and then integrate with the traditional model to improve the overall performance of the recommendation system. This will not change the existing recommendation framework in the short term, and can make the most of the technical advantages of deep learning. Google, linkedin and others have started to use the neural language model to extract text features so that they can better express text messages. For example, audio and video recommendation problems, YouTube uses deep learning to extract video features and effectively recommend users' favorite video.

The second is to use deep learning techniques to solve problems that were not easy to solve before. For example, the famous music broadcasting platform, Spotify, uses a recursive neural network to predict the next recommended song, which has a good effect. The recursive neural network is characterized by the ability to express long distance dependencies naturally in the model, while the traditional method of matrix decomposition is more difficult to grasp such characteristics. With the success of AlphaGo, deep reinforcement learning has become a popular research direction.

True artificial intelligence not only need to be more flexible and powerful model, but also need the researchers category to have a further thinking on the issue of recommendation systems, and consider how to optimize the user, system, market overall relations. For example, on the e-commerce site, users want to buy the right things conveniently, but also want to know about fashion information, or communicate with friends who have similar interests. The recommendation system of single target optimization cannot meet the complex requirements. How to excavate the potential demand of users and how to optimize the overall recommendation system.

\section{Summary}

Artificial intelligence technology has great influence on the recommendation system. From the artificial recommendation to the deep learning model, the recommendation system has become the cornerstone of many Internet applications. Research and development over the past decade has driven the development of artificial intelligence in more directions. For now, the recommendation system has a long way to go to become an intelligent system. We need to stand in a higher dimension to look at and consider recommendation system, and put forward more challenging problems, it can lead us to reform the methodology on the basis of these problems, and to establish a set of recommendation system theory and practice which can greatly enrich the user's experience and improve the product's value.

\section{Acknowledgements}

This research is supported by National Science-technology Support Plan Projects (NO. 2015BAD29B01), Gansu Provincial Higher Education Research Project (No.2015B-007), Gansu Province Education Science Planning Project ( No.GS[2016]GHB0203).

\section{Reference}

[1] Rubén González Crespo, Oscar Sanjuán Martínez, Juan Manuel Cueva Lovelle , B. Cristina Pelayo García-Bustelo, José Emilio Labra Gayo, Patricia Ordoñez de Pablos . Recommendation 
System based on user interaction data applied to intelligent electronic books[J]. Computers in Human Behavior,2010,27(4).

[2] Yiyang Zhang, Jianxin (Roger) Jiao. An associative classification-based recommendation system for personalization in B2C e-commerce applications[J]. Expert Systems With Applications,2006,33(2).

[3] Jian Wei,Jianhua He, Kai Chen,Yi Zhou,Zuoyin Tang. Collaborative filtering and deep learning based recommendation system for cold start items[J]. Expert Systems With Applications,2017,69.

[4] Sangwook Kim, Jehan Jung, Swathi Kavuri, Minho Lee. Intention Estimation and Recommendation System Based on Attention Sharing[M].Springer Berlin Heidelberg:2013-0615.

[5] Do-Eun Cho, Sang-Soo Yeo, Si Jung Kim. An Adaptive Intelligent Recommendation Scheme for Smart Learning Contents Management Systems[M].Springer Netherlands:2013-06-15.

[6] Marcos Martín Pozo, José Antonio Iglesias, Agapito Ismael Ledezma. Intelligent Promotions Recommendation System for Instaprom Platform[M].Springer International Publishing:201406-15.

[7] Timothy K. Shih, Chuan-Feng Chiu, Hui-huang Hsu, Fuhua Lin. An integrated framework for recommendation systems in e-commerce[J]. Industrial Management \&amp; Data Systems,2002,102(8).

[8] Yan Guo, Minxi Wang, Xin Li. Application of an improved Apriori algorithm in a mobile ecommerce recommendation system[J]. Industrial Management \&amp; Data Systems,2017,117(2).

[9] Yi Wang, Hao Yuan Ou, Jian Ming Zhang. Design and Implementation of E-Commerce Recommendation System Based on Ontology Technology[J]. Advanced Materials Research,2014,3321(978).

[10] Meng Han. The Design and Implementation of E-Commerce Personalized Services Based on Collaborative Filtering Recommendation System[J]. Applied Mechanics and Materials,2014,3634(687).

[11] Meziane, Farid. Artificial Intelligence Applications for Improved Software Engineering Development: New Prospects[M].IGI Global:2009-06-15.

[12] Jacek Grudzień. The Development of Computer Competencies Using Artificial Intelligence[J]. International Journal of Information and Communication Technologies in Education,2014,3(1). 